\title{
On the Correlation of Increasing Families
}

\author{
Gil Kalai*, Nathan Keller ${ }^{\dagger}$, and Elchanan Mossel ${ }^{\ddagger}$
}

October 11, 2018

\begin{abstract}
The classical correlation inequality of Harris asserts that any two monotone increasing families on the discrete cube are nonnegatively correlated. In 1996, Talagrand [19] established a lower bound on the correlation in terms of how much the two families depend simultaneously on the same coordinates. Talagrand's method and results inspired a number of important works in combinatorics and probability theory.

In this paper we present stronger correlation lower bounds that hold when the increasing families satisfy natural regularity or symmetry conditions. In addition, we present several new classes of examples for which Talagrand's bound is tight.

A central tool in the paper is a simple lemma asserting that for monotone events noise decreases correlation. This lemma gives also a very simple derivation of the classical FKG inequality for product measures, and leads to a simplification of part of Talagrand's proof.
\end{abstract}

\section{Introduction}

Definition 1.1. Let $\Omega_{n}$ denote the discrete cube $\{0,1\}^{n}$, and identify elements of $\Omega_{n}$ with subsets of $[n]=\{1,2, \ldots, n\}$ in the natural manner. A family $\mathcal{A} \subset \Omega_{n}$ is increasing if $(S \in$ $\mathcal{A}) \wedge\left(S \subset T\right.$ ) implies $T \in \mathcal{A}$ (alternatively, if the characteristic function $\mathbf{1}_{\mathcal{A}}$ is non-decreasing with respect to the natural partial order on $\Omega_{n}$ ).

One of the best-known correlation inequalities is Harris' inequality [10] which asserts that any two increasing families $\mathcal{A}, \mathcal{B} \subset \Omega_{n}$ are nonnegatively correlated, i.e., satisfy

$$
\operatorname{Cov}(\mathcal{A}, \mathcal{B})=\mu(\mathcal{A} \cap \mathcal{B})-\mu(\mathcal{A}) \mu(\mathcal{B}) \geq 0
$$

where $\mu$ is the uniform measure on $\Omega_{n}$. In 1996, Talagrand [19] presented a lower bound on the correlation, in terms of influences of the variables on $\mathcal{A}, \mathcal{B}$.

Definition 1.2. The influence of the $k^{\text {th }}$ variable on $\mathcal{A} \subset \Omega_{n}$ is

$$
I_{k}(\mathcal{A})=2 \mu\left(\left\{x \in \mathcal{A} \mid x \oplus e_{k} \notin \mathcal{A}\right\}\right),
$$

where $x \oplus e_{k}$ is gotten from $x$ by replacing $x_{k}$ by $1-x_{k}$. The total influence of $\mathcal{A}$ is $I(\mathcal{A})=$ $\sum_{k=1}^{n} I_{k}(\mathcal{A})$.

${ }^{*}$ Einstein Institute of Mathematics, Hebrew University, Jerusalem, Israel. kalai@math.huji.ac.il. Research supported by ERC advanced grant 320924, BSF grant 2014290, and NSF grant DMS-1300120.

${ }^{\dagger}$ Department of Mathematics, Bar Ilan University, Ramat Gan, Israel. nathan.keller27@gmail.com. Research supported by the Israel Science Foundation (grant no. 402/13), the Binational US-Israel Science Foundation (grant no. 2014290), and by the Alon Fellowship.

${ }^{\ddagger}$ Department of Statistics, University of Pennsylvania, 3730 Walnut Street, Philadelphia, PA 19104 and Departments of Statistics and Computer Science, U.C. Berkeley, 367 Evans Hall, Berkeley CA 94720. mossel@wharton. upenn.edu. Research supported by NSF grant CCF 1320105, DOD ONR grant N00014-141-0823, and grant 328025 from the Simons Foundation. 
We also write $\mathcal{W}_{1}(\mathcal{A}, \mathcal{B})=\sum_{i=1}^{n} I_{i}(\mathcal{A}) I_{i}(\mathcal{B})$.

Theorem 1.3 (Talagrand). Let $\mathcal{A}, \mathcal{B} \subset \Omega_{n}$ be increasing. Then

$$
\operatorname{Cov}(\mathcal{A}, \mathcal{B}) \geq c \sum_{i=1}^{n} \frac{I_{i}(\mathcal{A}) I_{i}(\mathcal{B})}{\log \left(e / \sum_{i=1}^{n} I_{i}(\mathcal{A}) I_{i}(\mathcal{B})\right)}=c \varphi\left(\mathcal{W}_{1}(\mathcal{A}, \mathcal{B})\right)
$$

where $\varphi(x)=x / \log (e / x)$ and $c$ is a universal constant.

Talagrand's theorem and the central lemma used in its proof (Lemma 2.7 below) were used in several subsequent works in combinatorics and probability theory (e.g., [1, 9, 13, 20]), most notably in the BKS noise sensitivity theorem [2].

So far, only two classes of tightness examples for Talagrand's lower bound are known. Talagrand [19] showed that his lower bound is tight when $\mathcal{A}, \mathcal{B}$ are increasing Hamming balls, i.e., have the form $\left\{x: \sum x_{i}>t\right\}$, where the thresholds $t_{\mathcal{A}}$ and $t_{\mathcal{B}}$ are chosen such that $\mu(\mathcal{A})=\epsilon$ and $\mu(\mathcal{B})=1-\epsilon$. In [11], the second author presented another example, based on Ben-Or and Linial's tribes function [3], defined as follows. Partition $[n]$ into $n / r$ disjoint sets $T_{1}, \ldots, T_{n / r}$ of $r$ elements, where $r \approx \log n-\log \log n$, and define $\mathcal{A}$ by setting $x \in \mathcal{A}$ iff there exists $j$ such that $x_{i}=1$ for all $i \in T_{j}$. Let $\mathcal{B}$ be the dual family of $\mathcal{A}$, i.e., $x=\left(x_{1}, \ldots, x_{n}\right) \in \mathcal{B}$ iff $\bar{x}=\left(1-x_{1}, \ldots, 1-x_{n}\right) \notin \mathcal{A}$. Then (11) is tight for $\mathcal{A}, \mathcal{B}$.

While the two examples seem dissimilar, they share a central common feature: in both examples, $I_{i}(\mathcal{A})=I_{i}(\mathcal{B})$ for all $i \in[n]$. (Moreover, in both cases $\mathcal{B}$ is the dual of $\mathcal{A}$.) Thus, a first motivation of the current paper is seeking to find other tightness examples, especially examples in which the relation between the structures of $\mathcal{A}$ and $\mathcal{B}$ is not so strong.

A second motivation is an alternative correlation lower bound, proved recently by Keller, Mossel, and Sen [14].

Theorem 1.4. Let $\mathcal{A}, \mathcal{B} \subset \Omega_{n}$ be increasing. Then

$$
\operatorname{Cov}(\mathcal{A}, \mathcal{B}) \geq c \sum_{i=1}^{n} \frac{I_{i}(\mathcal{A})}{\sqrt{\log \frac{e}{I_{i}(\mathcal{A})}}} \frac{I_{i}(\mathcal{B})}{\sqrt{\log \frac{e}{I_{i}(\mathcal{B})}}}=c \sum_{i=1}^{n} \psi\left(I_{i}(A)\right) \psi\left(I_{i}(B)\right)
$$

where $\psi(x)=x / \sqrt{\log (e / x)}$ and $c$ is a universal constant.

It turns out that neither of the lower bounds is strictly stronger than the other. While Talagrand's bound is better for $\mathcal{A}$ being a small Hamming ball and $\mathcal{B}$ being its dual, there are cases of interest for which (2) is better. E.g., for $\mathcal{A}$ being a small Hamming ball and $\mathcal{B}$ being the "majority" (i.e., $\left\{x: \sum x_{i}>n / 2\right\}$ ), (2) is always stronger, and may be stronger even by a multiplicative factor of $\sqrt{n / \log n}$. Hence, it is tempting to find an improved lower bound that will combine the advantages of (11) and (2).

Our main result is such a "combined" lower bound that holds under a weak regularity assumption on the families.

Definition 1.5. A family $\mathcal{A} \subset \Omega_{n}$ is regular if all its influences are equal.

Note that in all examples mentioned so far (and actually, in most examples in the field, except for "dictatorships" and "juntas"), both $\mathcal{A}$ and $\mathcal{B}$ are regular. In fact, in those cases the families are weakly symmetric, namely invariant under a transitive group of permutations on the variables. 
Definition 1.6. Two increasing families $\mathcal{A}$ and $\mathcal{B}$ are similar if all ratios $I_{i}(\mathcal{A}) / I_{i}(\mathcal{B})$ are equal, and weakly similar if for some $c^{\prime}>0, \max \left\{I_{i}(\mathcal{A}) / I_{i}(\mathcal{B})\right\} \leq c^{\prime} \min \left\{I_{i}(\mathcal{A}) / I_{i}(\mathcal{B})\right\}$. Of course, all regular families are mutually similar.

Theorem 1.7. Let $\mathcal{A}, \mathcal{B} \subset \Omega_{n}$ be increasing and similar. Then

$$
\operatorname{Cov}(\mathcal{A}, \mathcal{B}) \geq c \frac{\mathcal{W}_{1}(\mathcal{A}, \mathcal{B})}{\sqrt{\log \frac{e}{W_{1}(\mathcal{A}, \mathcal{A})}} \sqrt{\log \frac{e}{W_{1}(\mathcal{B}, \mathcal{B})}}},
$$

where $c>0$ is a universal constant.

In particular, if $\mathcal{A}, \mathcal{B} \subset \Omega_{n}$ are regular, then

$$
\operatorname{Cov}(\mathcal{A}, \mathcal{B}) \geq c \sum_{i=1}^{n} \frac{I_{i}(\mathcal{A})}{\sqrt{\log \frac{e}{n I_{i}(\mathcal{A})^{2}}}} \frac{I_{i}(\mathcal{B})}{\sqrt{\log \frac{e}{n I_{i}(\mathcal{B})^{2}}}}=c \frac{I(\mathcal{A}) I(\mathcal{B})}{n \sqrt{\log \frac{e n}{I(\mathcal{A})^{2}}} \sqrt{\log \frac{e n}{I(\mathcal{B})^{2}}}} .
$$

When we let $c$ depend on $c^{\prime}$, the theorem extends to weakly similar increasing families $\mathcal{A}$ and $\mathcal{B}$. It is easy to show (see Claim 3.1 ) that (3) is always at least as strong as both (11) and (2). Moreover, in some cases of interest it is strictly stronger. For example, in the case of $\mathcal{A}$ being a Hamming ball with $\mu(\mathcal{A})=O(1 / n)$ and $\mathcal{B}$ being the "majority", (3) is tight, while (2) is off by a factor of $\sqrt{\log n}$, and (1) is off by $\sqrt{\log (1 / \mu(\mathcal{A}))}$, that may be as large as $\sqrt{n}$.

We achieve Theorem 1.7 by proposing a somewhat simpler proof of Theorem 1.3 that allows to handle better similarity and regularity assumptions on the families. The new proof uses a simple lemma regarding a property of the classical noise operator.

Definition 1.8. Let $0 \leq \rho \leq 1$. The noise operator $T_{\rho}: \mathbb{R}^{\Omega_{n}} \rightarrow \mathbb{R}^{\Omega_{n}}$ is defined by

$$
T_{\rho} f(x)=\mathbb{E}\left[f\left(N_{\rho} x\right)\right],
$$

where $N_{\rho}(x)$ is obtained from $x$ by leaving each coordinate of $x$ unchanged with probability $\rho$ and replacing it by a random value with probability $1-\rho$.

Lemma 1.9. Let $f, g: \Omega_{n} \rightarrow \mathbb{R}$ be increasing. Then the function $\rho \mapsto\left\langle T_{\rho} f, g\right\rangle$ (where $\langle\cdot, \cdot\rangle$ is the usual inner product on $\left.\left(\Omega_{n}, \mu\right)\right)$ is non-decreasing.

Lemma 1.9 is of independent interest. For example, it yields an instant proof of the FKG correlation inequality [8] for product measures. Indeed, as $\left\langle T_{0} f, g\right\rangle=\mathbb{E}[f] \mathbb{E}[g]$ and $\left\langle T_{1} f, g\right\rangle=$ $\mathbb{E}[f g]$, we immediately obtain

$$
\operatorname{Cov}(f, g):=\mathbb{E}[f g]-\mathbb{E}[f] \mathbb{E}[g] \geq 0 .
$$

A consequence of Theorem 1.7 is:

Corollary 1.10. If $\mathcal{A}$ is increasing, regular and balanced and $\mathcal{B}$ is the majority function, then

$$
\operatorname{Cov}(\mathcal{A}, \mathcal{B}) \geq c \sqrt{\log n} / \sqrt{n}
$$

where $c>0$ is a universal constant.

We note that both Theorems 1.3 and 1.4 give a weaker lower bound of $c / \sqrt{n}$. On the other hand, we can show that when $\mathcal{A}$ is the tribes family, then $\operatorname{Cov}(\mathcal{A}, \mathrm{MAJ})=\Theta(\log n / \sqrt{n})$, and we conjecture that this lower bound holds in general. Furthermore, we conjecture that the following holds: 
Conjecture 1.11. If $\mathcal{A}$ is increasing and balanced then there exists an increasing $\mathcal{B}$ represented by a linear threshold function (i.e., $\mathcal{B}=\left\{x: \sum a_{i} x_{i}>t\right\}$ for nonnegative weights $a_{i}$ ), such that $\operatorname{Cov}(\mathcal{A}, \mathcal{B}) \geq c \log n / \sqrt{n}$, for a universal constant $c$.

Our next result gives a hybrid of the bounds (11) and (2), under a strong symmetry condition on only one of the families $\mathcal{A}, \mathcal{B}$.

Definition 1.12. A family $\mathcal{A} \subset \Omega_{n}$ is fully symmetric if it is invariant under the action of $\mathbb{S}_{n}$.

For example, while the Hamming balls considered above are fully symmetric, the tribes functions are only weakly symmetric but not fully symmetric.

Theorem 1.13. Let $\mathcal{A} \subset \Omega_{n}$ be increasing and $\mathcal{B} \subset \Omega_{n}$ be increasing and fully symmetric. Then

$$
\operatorname{Cov}(\mathcal{A}, \mathcal{B}) \geq c_{1} \sum_{i=1}^{n} \frac{I_{i}(\mathcal{A})}{\sqrt{\log \frac{e}{I_{i}(\mathcal{A})}}} \frac{I_{i}(\mathcal{B})}{\sqrt{\log \frac{e^{2}}{\mathcal{W}_{1}(\mathcal{B}, \mathcal{B})}}} \geq c_{2} \frac{\mu(\mathcal{B})(1-\mu(\mathcal{B}))}{\sqrt{n}} \sum_{i=1}^{n} \frac{I_{i}(\mathcal{A})}{\sqrt{\log \frac{e}{I_{i}(\mathcal{A})}}}
$$

where $c_{1}, c_{2}$ are universal constants.

It can be seen that (6) is always stronger than (2), but sometimes weaker than (1). In particular, it is tight for the correlation of a small Hamming ball and the "majority" family considered above. The proof of Theorem 1.13 follows Talagrand's original proof, with an enhancement that allows to handle different assumptions on $\mathcal{A}, \mathcal{B}$ in a better way. Without additional assumptions on $\mathcal{A}$ and $\mathcal{B}$, the proof techniques of Theorem 1.13 give a new proof of Theorem 1.4.

Finally, we prove simple sufficient conditions for tightness of Talagrand's lower bound (1), and use them to show that (11) is tight for several new examples, including (among others) $\mathcal{A}$ representing an increasing linear threshold function with low influences and $\mathcal{B}$ being $\mathcal{A}$ 's dual.

All our results extend verbatim to bounded functions $f: \Omega_{n} \rightarrow[-1,1]$, with influences defined as

$$
I_{k}(f)=\mathbb{E}\left[\left|f(x)-f\left(x \oplus e_{k}\right)\right|\right] .
$$

For sake of completeness, in the following sections we prove our results in the more general form 1

This paper is organized as follows. In Section 2 we present the proof of Lemma 1.9 and use it to simplify the proof of Theorem 1.3. In Section 3 we present the proof of Theorem 1.7 along with some examples showing that the similarity conditions are necessary. The (more involved) proof of Theorem 1.13 is given in Section 4. Section 5 features new tightness examples of Talagrand's lower bound. We conclude the paper with a few open problems in Section 6 .

\section{Noise and Correlation}

\subsection{Preliminaries}

Notation 2.1. For $x, y \in \Omega_{n}$ we write $x \leq y$ if $x_{i} \leq y_{i}$ for all $i$. A function $f: \Omega_{n} \rightarrow \mathbb{R}$ is called increasing or monotone if $f(x) \leq f(y)$ whenever $x \leq y$.

\footnotetext{
${ }^{1}$ We note that there are several alternative generalizations of the notion of influences to general functions on $\Omega_{n}$ (see, e.g., 7] and the references therein).
} 
The main technical tool used in this paper, as well as in Talagrand's work, is the Fourier-Walsh expansion.

Definition 2.2. Let $f: \Omega_{n} \rightarrow \mathbb{R}$. The Fourier-Walsh expansion of $f$ is the unique expansion

$$
f=\sum_{S \subset[n]} \alpha_{S} u_{S},
$$

where for $T \subset[n]$,

$$
u_{S}(T)=(-1)^{|S \cap T|} .
$$

The coefficients $\alpha_{S}$ are also denoted by $\hat{f}(S)$, and the level of the coefficient $\hat{f}(S)$ is $|S|$.

Since $\left\{u_{S}\right\}_{S \subset[n]}$ is an orthonormal basis for the function space $\mathbb{R}^{\Omega_{n}}$ (relative to the usual inner product $\langle\cdot, \cdot\rangle$ with respect to uniform measure), the representation is indeed unique, with $\hat{f}(S)=$ $\left\langle f, u_{S}\right\rangle$, and we have Parseval's identity:

$$
\langle f, g\rangle=\sum \hat{f}(S) \hat{g}(S) \quad \forall f, g .
$$

The noise operator $T_{\rho}$ has a simple representation in terms of the Fourier-Walsh expansion: For any $f=\sum_{S} \hat{f}(S) u_{S}$ and $\rho \in[0,1]$, we have

$$
T_{\rho} f=\sum_{S} \rho^{|S|} \hat{f}(S) u_{S}
$$

A standard operator we consider is the $i^{\text {th }}$ discrete derivative:

Definition 2.3. For $i \in[n]$, define $\Delta_{i}: \mathbb{R}^{\Omega_{n}} \rightarrow \mathbb{R}^{\Omega_{n}}$ by $\Delta_{i} f(x)=\frac{1}{2}\left[f(x)-f\left(x \oplus e_{i}\right)\right]$.

It is easy to see that the Fourier expansion of $\Delta_{i} f$ is

$$
\Delta_{i} f=\sum_{S \ni i} \hat{f}(S) u_{S}
$$

We use the following basic properties of the Fourier expansion and the noise operator:

Claim 2.4. (a) For any $f, g: \Omega_{n} \rightarrow \mathbb{R}$, we have $\operatorname{Cov}(f, g)=\sum_{S \neq \emptyset} \hat{f}(S) \hat{g}(S)$ (this follows immediately from (77), since $\hat{f}(\emptyset) \hat{g}(\emptyset)=\mathbb{E}[f] \mathbb{E}[g]$ ).

(b) For any increasing $f: \Omega_{n} \rightarrow \mathbb{R}$ and any $\rho \in[0,1], T_{\rho} f$ is increasing (see, e.g., [12, Proof of Proposition 4.4]).

(c) For any increasing $f: \Omega_{n} \rightarrow[-1,1]$ and any $i \in[n], I_{i}(f)=\hat{f}(\{i\})$ (this follows immediately from the definitions). As a result, $\sum_{i} I_{i}(f)^{2}=\sum_{i} \hat{f}(\{i\})^{2} \leq 1$ by (7).

For more background on the Fourier-Walsh expansion the reader is referred to [16]. 


\subsection{Noise decreases correlation}

We present two proofs of Lemma 1.9, which essentially shows that application of noise reduces the correlation of increasing functions. The first proof uses the Fourier-Walsh expansion of the noise operator, while the second uses only the chain rule and resembles the simple proof of Russo's lemma [18]. Recall the formulation of the Lemma:

Lemma. Let $f, g: \Omega_{n} \rightarrow \mathbb{R}$ be increasing. Then the function $h(\rho)=\left\langle T_{\rho} f, g\right\rangle$ is non-decreasing.

First Proof. First, we note that since for any decomposition $\rho=\rho_{1} \cdot \rho_{2}$ we have $T_{\rho} f=$ $T_{\rho_{2}}\left(T_{\rho_{1}} f\right)$, and since $T_{\rho^{\prime}} f$ is increasing for any $\rho^{\prime}$ (Claim 2.4(b)), it is sufficient to show that $h^{\prime}(1)$ is nonnegative. By (7) and (8), we have

$$
h(\rho)=\left\langle T_{\rho} f, g\right\rangle=\sum_{S} \rho^{|S|} \hat{f}(S) \hat{g}(S),
$$

and thus,

$$
h^{\prime}(1)=\sum_{S}|S| \hat{f}(S) \hat{g}(S)=\sum_{i} \sum_{S \ni i} \hat{f}(S) \hat{g}(S)=\sum_{i}\left\langle\Delta_{i} f, \Delta_{i} g\right\rangle,
$$

the last equality using (7) once again. This completes the proof, as $\Delta_{i} f(x) \cdot \Delta_{i} g(x) \geq 0$ for any $x$ by the monotonicity of $f, g$.

Second Proof. Define an "asymmetric" noise operator $T_{\rho_{1}, \ldots, \rho_{n}}$ by

$$
T_{\rho_{1}, \ldots, \rho_{n}} f(x)=\mathbb{E}\left[f\left(N_{\rho_{1}, \ldots, \rho_{n}} x\right)\right],
$$

where $N_{\rho_{1}, \ldots, \rho_{n}}(x)$ is obtained from $x$ by leaving the $i$ 'th coordinate of $x$ unchanged with probability $\rho_{i}$ and replacing it by a random value with probability $1-\rho_{i}$. As $h(\rho)=\left\langle T_{\rho, \rho, \ldots, \rho} f, g\right\rangle$, we can apply the chain rule to assert

$$
h^{\prime}(1)=\left.\sum_{i=1}^{n} \frac{\partial}{\partial \rho_{i}}\left\langle T_{\rho_{1}, \ldots, \rho_{n}} f, g\right\rangle\right|_{\left(\rho_{1}, \ldots, \rho_{n}\right)=(1, \ldots, 1)}=\sum_{i}\left\langle\Delta_{i} f, \Delta_{i} g\right\rangle .
$$

The rest of the argument is the same as in the first proof.

Remark 2.5. Note that as $\mathbb{E}\left[T_{\rho} f\right]=\mathbb{E}[f]$, an equivalent formulation of Lemma 1.9 is that the function $\rho \mapsto \operatorname{Cov}\left(T_{\rho} f, g\right)$ is non-decreasing. This formulation will be used in the proof of Theorem 1.3 below.

\subsection{A simpler proof of Talagrand's inequality}

Notation 2.6. From now on, for $f, g: \Omega_{n} \rightarrow \mathbb{R}$ and $d \in \mathbb{N}$, we denote $\mathcal{W}_{d}(f, g)=\sum_{|S|=d} \hat{f}(S) \hat{g}(S)$ and $\mathcal{W}_{d}(f)=\mathcal{W}_{d}(f, f)$. Note that for increasing $\mathcal{A}$ and $\mathcal{B}, \mathcal{W}_{1}\left(1_{\mathcal{A}}, 1_{\mathcal{B}}\right)=\sum_{i} I_{i}(\mathcal{A}) I_{i}(\mathcal{B})$.

A generalized formulation of Theorem 1.3 (using Claim 2.4(c)) is the following:

Theorem. Let $f, g: \Omega_{n} \rightarrow[-1,1]$ be increasing. Then

$$
\operatorname{Cov}(f, g) \geq c \mathcal{W}_{1}(f, g)\left(\log \left(e / \mathcal{W}_{1}(f, g)\right)\right)^{-1},
$$

where $c$ is a universal constant.

The original proof of Theorem 1.3 presented in [19] consists of two parts. The first part which is more complex and which we keep virtually unchanged, is proving the following lemma, which bounds the second-level Fourier-Walsh coefficients of $f, g$ in terms of the first-level ones: 
Lemma 2.7 (Talagrand). Let $f, g: \Omega_{n} \rightarrow[-1,1]$ be increasing. Then

$$
\mathcal{W}_{2}(f, g) \leq c \mathcal{W}_{1}(f, g) \log \left(e / \mathcal{W}_{1}(f, g)\right)
$$

where $c$ is a universal constant.

This lemma appears to be of independent interest, and probably has more applications than Theorem 1.3. A somewhat simpler (but still rather complex) proof of the lemma is given in [13, along with some generalizations.

Our argument uses the following generalization of Lemma 2.7. proved in [2] (a qualitative version) and in [13] (a quantitative version).

Lemma 2.8. For all increasing $f, g: \Omega_{n} \rightarrow[-1,1]$, and for all $2 \leq d \leq \log \left(e / \mathcal{W}_{1}(f, g)\right) / 2$,

$$
\mathcal{W}_{d}(f, g) \leq \frac{5 e}{d}\left(\frac{2 e}{d-1}\right)^{d-1} \mathcal{W}_{1}(f, g)\left(\log \left(d / \mathcal{W}_{1}(f, g)\right)\right)^{d-1}
$$

The proof of Lemma 2.8 is essentially the same as the proof of Lemma 2.7. To simplify notations, we denote $C(d)=\frac{5 e}{d}\left(\frac{2 e}{d-1}\right)^{d-1}$, as in the sequel we use only the fact that $C(d)=$ $O(1)$.

The second part of Talagrand's proof, is a somewhat complex inductive argument that deduces the theorem from Lemma 2.7. We show now that the inductive argument in the second part of Talagrand's proof can be replaced with a very simple argument, using Lemma 1.9.

Before presenting the proof, we explain the main idea behind it, which is quite different from the ideas used in Talagrand's proof. By Claim 2.4(a), the correlation can be expressed in terms of the Fourier-Walsh coefficients as $\operatorname{Cov}(f, g)=\sum_{S \neq \emptyset} \hat{f}(S) \hat{g}(S)$. By Claim 2.4(c), all first-level terms in the right hand side (i.e., all terms with $|S|=1$ ) are nonnegative and their total contribution is $\mathcal{W}_{1}(f, g)$. The other terms may be negative, and the assertion of the theorem is that they cannot be "too negative", in the sense that their total contribution is bounded from below by $-\mathcal{W}_{1}(f, g)+c \mathcal{W}_{1}(f, g)\left(\log \left(e / \mathcal{W}_{1}(f, g)\right)\right)^{-1}$. Hence, our goal is to bound from below the contribution of all levels $d \geq 2$.

To obtain this, we use the noise operator $T_{\rho}$ whose application suppresses the high-level coefficients. By replacing $\operatorname{Cov}(f, g)$ with $\operatorname{Cov}\left(T_{\rho} f, g\right)$ for an appropriate choice of $\rho$, we obtain an expression $\sum_{S \neq \emptyset} \rho^{|S|} \hat{f}(S) \hat{g}(S)$ in which the (possibly negative) contribution of all levels $d \geq 2$ is dominated by the positive contribution of the first level. Lemma 1.9 then allows to go back to $\operatorname{Cov}(f, g)$.

Proof of Theorem 1.3. Let $\rho=c_{0}\left(\log \left(e / \mathcal{W}_{1}(f, g)\right)\right)^{-1}$, where $c_{0}$ is a sufficiently small constant. By Claim 2.4(a) and Equation (8),

$$
\begin{aligned}
\operatorname{Cov}\left(T_{\rho} f, g\right) & =\sum_{S \neq \emptyset} \rho^{|S|} \hat{f}(S) \hat{g}(S)=\sum_{d \geq 1} \rho^{d} \mathcal{W}_{d}(f, g)= \\
& =\rho \mathcal{W}_{1}(f, g)+\sum_{2 \leq d \leq \log \left(e / \mathcal{W}_{1}(f, g)\right) / 2} \rho^{d} \mathcal{W}_{d}(f, g)+\sum_{d>\log \left(e / \mathcal{W}_{1}(f, g)\right) / 2} \rho^{d} \mathcal{W}_{d}(f, g) .
\end{aligned}
$$

By Lemma 2.8, for every $2 \leq d \leq \log \left(e / \mathcal{W}_{1}(f, g)\right) / 2$ we have

$$
\rho^{d} \mathcal{W}_{d}(f, g) \leq \rho^{d} C(d) \mathcal{W}_{1}(f, g)\left(\log \left(d / \mathcal{W}_{1}(f, g)\right)\right)^{d-1} \leq 2^{-d} \rho \mathcal{W}_{1}(f, g),
$$


where the last inequality holds by the choice of $\rho$ (here is where $c_{0}$ should be taken sufficiently small). For any $d>\log \left(e / \mathcal{W}_{1}(f, g)\right) / 2$, we use the bound

$$
\rho^{d} \mathcal{W}_{d}(f, g) \leq \rho^{d}<2^{-d} \rho \mathcal{W}_{1}(f, g)
$$

Combining, we get

$$
\sum_{d \geq 2}\left|\rho^{d} \mathcal{W}_{d}(f, g)\right| \leq \sum_{d \geq 2} 2^{-d} \rho \mathcal{W}_{1}(f, g) \leq \rho \mathcal{W}_{1}(f, g) / 2
$$

Hence,

$$
\operatorname{Cov}\left(T_{\rho} f, g\right)=\rho \mathcal{W}_{1}(f, g)+\sum_{d \geq 2} \rho^{d} \mathcal{W}_{d}(f, g) \geq \rho \mathcal{W}_{1}(f, g) / 2=c^{\prime} \mathcal{W}_{1}(f, g)\left(\log \left(e / \mathcal{W}_{1}(f, g)\right)\right)^{-1}
$$

Therefore, by Lemma 1.9,

$$
\operatorname{Cov}(f, g) \geq \operatorname{Cov}\left(T_{\rho} f, g\right) \geq c^{\prime} \mathcal{W}_{1}(f, g)\left(\log \left(e / \mathcal{W}_{1}(f, g)\right)\right)^{-1},
$$

as asserted.

Remark 2.9. We stress that the new proof replaces only the inductive part of Talagrand's proof. The main part of the proof (i.e., the proof of Lemma 2.7) remains unchanged.

\section{Improved Correlation Bound Under Similarity}

In this section we present the proof of Theorem 1.7, and demonstrate by several examples that the similarity assumption in the theorem is essential.

\subsection{Proof of Theorem 1.7}

A generalized statement of Theorem 1.7 (using Claim 2.4(c) once again) is:

Theorem. Let $f, g: \Omega_{n} \rightarrow[-1,1]$ be increasing and similar. Then

$$
\operatorname{Cov}(\mathrm{f}, \mathrm{g}) \geq c \mathcal{W}_{1}(f, g)\left(\log \left(e / \mathcal{W}_{1}(f)\right)\right)^{-1 / 2}\left(\log \left(e / \mathcal{W}_{1}(g)\right)\right)^{-1 / 2},
$$

where $c$ is a universal constant.

We note that the lower bound of Theorem 1.7 is stronger (up to a constant) than the bounds of Theorems 1.3 and 1.4 :

Claim 3.1. Let $f, g: \Omega_{n} \rightarrow[-1,1]$ be increasing. Then

(a) $\mathcal{W}_{1}(f, g)\left(\log \left(e / \mathcal{W}_{1}(f)\right)\right)^{-1 / 2}\left(\log \left(e / \mathcal{W}_{1}(g)\right)\right)^{-1 / 2} \geq \mathcal{W}_{1}(f, g)\left(\log \left(e / \mathcal{W}_{1}(f, g)\right)^{-1}\right.$,

(b) $\mathcal{W}_{1}(f, g)\left(\log \left(e / \mathcal{W}_{1}(f)\right)\right)^{-1 / 2}\left(\log \left(e / \mathcal{W}_{1}(g)\right)\right)^{-1 / 2} \geq 0.5 \sum_{i=1}^{n} \frac{I_{i}(f)}{\sqrt{\log \frac{e}{I_{i}(f)}}} \frac{I_{i}(g)}{\sqrt{\log \frac{e}{I_{i}(g)}}}$.

Proof. For (a), as the numerators are equal, it is sufficient to compare the denominators. We have

$$
\begin{aligned}
\log \left(e / \mathcal{W}_{1}(f, g)\right) \geq \log \left(\frac{e}{\sqrt{\mathcal{W}_{1}(f)} \sqrt{\mathcal{W}_{1}(g)}}\right) & =\frac{1}{2}\left(\log \left(\frac{e}{\mathcal{W}_{1}(f)}\right)+\log \left(\frac{e}{\mathcal{W}_{1}(g)}\right)\right) \\
& \geq \sqrt{\log \left(e / \mathcal{W}_{1}(f)\right)} \sqrt{\log \left(e / \mathcal{W}_{1}(g)\right)}
\end{aligned}
$$


where the first inequality uses Cauchy-Schwarz and the second uses the inequality between the arithmetic and geometric means.

The inequality (b) is immediate, as for any $i$ we have

$$
\sqrt{\log \frac{e}{I_{i}(f)}}=\sqrt{1 / 2} \sqrt{\log \frac{e^{2}}{I_{i}(f)^{2}}} \geq \sqrt{1 / 2} \sqrt{\log \left(e / \mathcal{W}_{1}(f)\right)}
$$

and similarly for $g$.

The strategy of the proof of (11) is similar to the simpler proof of Theorem 1.3 presented above, the only difference being the similarity assumption that allows applying Lemma 2.8 to $f$ and $g$ separately and then combining the results using the Cauchy-Schwarz inequality.

Proof of Theorem 1.7, Let

$$
\rho=c_{0}^{\prime}\left(\log \left(e / \mathcal{W}_{1}(f)\right)\right)^{-1 / 2}\left(\log \left(e / \mathcal{W}_{1}(g)\right)\right)^{-1 / 2},
$$

where $c_{0}^{\prime}$ is a sufficiently small constant. As in the proof of Theorem 1.3 above, we want to upper bound $\sum_{d \geq 2}\left|\rho^{d} \mathcal{W}_{d}(f, g)\right|$. By Cauchy-Schwarz, it is sufficient to bound

$$
\sum_{d \geq 2} \rho^{d} \sqrt{\mathcal{W}_{d}(f)} \sqrt{\mathcal{W}_{d}(g)}
$$

Applying the argument used above to obtain (10) to each of the functions $f, g$ separately (with $\rho_{f}=c_{f}\left(\log \left(e / \mathcal{W}_{1}(f)\right)\right)^{-1}$ and $\left.\rho_{g}=c_{g}\left(\log \left(e / \mathcal{W}_{1}(g)\right)\right)^{-1}\right)$, we obtain

$$
\rho_{f}^{d} \mathcal{W}_{d}(f) \leq 2^{-d} \rho_{f} \mathcal{W}_{1}(f) \quad \text { and } \quad \rho_{g}^{d} \mathcal{W}_{d}(g) \leq 2^{-d} \rho_{g} \mathcal{W}_{1}(g)
$$

for all $d \geq 2$. As $\sqrt{\rho_{f} \rho_{g}}=\sqrt{c_{f} c_{g}}\left(\log \left(e / \mathcal{W}_{1}(f)\right)\right)^{-1 / 2}\left(\log \left(e / \mathcal{W}_{1}(g)\right)\right)^{-1 / 2}=\rho$, we can combine the inequalities in (12) and sum over $d$ to get

$$
\sum_{d \geq 2} \rho^{d} \sqrt{\mathcal{W}_{d}(f)} \sqrt{\mathcal{W}_{d}(g)} \leq \sum_{d \geq 2} 2^{-d} \rho \sqrt{\mathcal{W}_{1}(f) \mathcal{W}_{1}(g)} \leq \rho \sqrt{\mathcal{W}_{1}(f) \mathcal{W}_{1}(g)} / 2
$$

By the similarity of $f$ and $g$, we have

$$
\sqrt{\mathcal{W}_{1}(f) \mathcal{W}_{1}(g)}=\mathcal{W}_{1}(f, g)
$$

and thus, (13) reads

$$
\sum_{d \geq 2} \rho^{d} \sqrt{\mathcal{W}_{d}(f) \mathcal{W}_{d}(g)} \leq \rho \mathcal{W}_{1}(f, g) / 2
$$

Subsequently,

$$
\begin{aligned}
\operatorname{Cov}\left(T_{\rho} f, g\right) & =\rho \mathcal{W}_{1}(f, g)+\sum_{d \geq 2} \rho^{d} \mathcal{W}_{d}(f, g) \geq \rho \mathcal{W}_{1}(f, g)-\sum_{d \geq 2} \rho^{d} \sqrt{\mathcal{W}_{d}(f) \mathcal{W}_{d}(g)} \geq \\
& \geq \rho \mathcal{W}_{1}(f, g) / 2=c \mathcal{W}_{1}(f, g)\left(\log \left(e / \mathcal{W}_{1}(f)\right)\right)^{-1 / 2}\left(\log \left(e / \mathcal{W}_{1}(g)\right)\right)^{-1 / 2}
\end{aligned}
$$

The assertion now follows from Lemma 1.9.

Remark 3.2. The proof applies almost without change if we only assume that $f, g$ are weakly similar (with respect to a constant $c^{\prime}$ ). The only change is that (14) holds only up to a multiplicative factor that depends on $c^{\prime}$, and that should be compensated by multiplying $\rho_{f}$ and $\rho_{g}$ by the same factor. As a result, (15) holds, with the constant $c$ depending on $c^{\prime}$. 


\subsection{A few counterexamples}

As the formulation of Theorem 1.7 makes sense for general increasing families, one could hope that it holds without the similarity assumption. The following examples indicate that this is not the case. In the examples, we denote by $m_{a}\left(x_{1}, \ldots, x_{\ell}\right)$ an increasing Hamming ball $\mathcal{C} \subset\{0,1\}^{\ell}$ with $\mu(\mathcal{C})=a$.

Example 3.1. For a small constant $a$, let $\mathcal{A}=m_{a}\left(x_{1}, \ldots, x_{n}\right)$, and let $\mathcal{B}=m_{1-a}\left(x_{1}, \ldots, x_{n}\right)$ be the dual of $\mathcal{A}$. A direct computation (see [19]) shows that Theorem 1.3 is tight for $(\mathcal{A}, \mathcal{B})$, as $\operatorname{Cov}(\mathcal{A}, \mathcal{B})=a^{2}$ and $\mathcal{W}_{1}\left(\mathbf{1}_{\mathcal{A}}, \mathbf{1}_{\mathcal{B}}\right)=\Theta\left(a^{2} \log (1 / a)\right)$, where $\Theta(\cdot)$ "hides" a constant factor independent of $a, n$ (the latter holds since $I_{i}(\mathcal{A})=I_{i}(\mathcal{B})=\Theta(a \sqrt{\log (1 / a)} / \sqrt{n})$ for all $\left.i\right)$.

Define $\mathcal{A}^{\prime}, \mathcal{B}^{\prime} \subset \Omega_{n+1}$ by

$$
\mathcal{A}^{\prime}=\left\{\left(x_{1}, \ldots, x_{n}, y\right):\left(\left(x_{1}, \ldots, x_{n}\right) \in \mathcal{A}\right) \vee(y=1)\right\}, \quad \text { and } \quad \mathcal{B}^{\prime}=m_{1-a}\left(x_{1}, \ldots, x_{n}, y\right) .
$$

We claim that the assertion of Theorem 1.7 does not hold for $\left(\mathcal{A}^{\prime}, \mathcal{B}^{\prime}\right)$.

The influences of $\mathcal{A}^{\prime}$ are $I_{i}\left(\mathcal{A}^{\prime}\right)=I_{i}(A) / 2$ for $i \in[n]$ and $I_{n+1}\left(\mathcal{A}^{\prime}\right)=1-a$, and the influences of $\mathcal{B}^{\prime}$ are $I_{i}\left(\mathcal{B}^{\prime}\right) \sim I_{i}(\mathcal{B})$ (where as usual $\alpha \sim \beta$ means $\alpha / \beta \rightarrow 1$ as $n \rightarrow \infty$ ). Hence,

$$
\mathcal{W}_{1}\left(\mathbf{1}_{\mathcal{A}^{\prime}}, \mathbf{1}_{\mathcal{B}^{\prime}}\right) \sim \sum_{i \leq n} I_{i}(\mathcal{A}) I_{i}(\mathcal{B}) / 2+\Theta(a \sqrt{\log (1 / a)} / \sqrt{n})=\Theta\left(a^{2} \log (1 / a)\right),
$$

while $\mathcal{W}_{1}\left(\mathbf{1}_{\mathcal{A}^{\prime}}\right)=\Theta(1)$ and $\mathcal{W}_{1}\left(\mathbf{1}_{\mathcal{B}^{\prime}}\right) \sim \mathcal{W}_{1}\left(\mathbf{1}_{\mathcal{B}}\right)=\Theta\left(a^{2} \log (1 / a)\right)$. Hence,

$$
\frac{\mathcal{W}_{1}\left(\mathbf{1}_{\mathcal{A}^{\prime}}, \mathbf{1}_{\mathcal{B}^{\prime}}\right)}{\sqrt{\log \left(1 / \mathcal{W}_{1}\left(\mathbf{1}_{\mathcal{A}^{\prime}}\right)\right)} \sqrt{\log \left(1 / \mathcal{W}_{1}\left(\mathbf{1}_{\mathcal{B}^{\prime}}\right)\right)}}=\Theta\left(a^{2} \sqrt{\log (1 / a)}\right)
$$

On the other hand, we claim that $\operatorname{Cov}\left(\mathcal{A}^{\prime}, \mathcal{B}^{\prime}\right) \sim a^{2}$. To see this, let $z$ be a new variable independent of all others, denote $\mathcal{B}^{\prime \prime}=m_{1-a}\left(x_{1}, \ldots, x_{n}, z\right)$, and consider $\mathcal{A}^{\prime}, \mathcal{B}^{\prime \prime}$ as subsets of $\{0,1\}^{n+2}$. As $\mathcal{A}^{\prime}$ does not depend on $z$ and $\mathcal{B}^{\prime \prime}$ does not depend on $y$, a direct computation yields $\operatorname{Cov}\left(\mathcal{A}^{\prime}, \mathcal{B}^{\prime \prime}\right) \sim \operatorname{Cov}(\mathcal{A}, \mathcal{B}) / 2=a^{2} / 2$. Since $\mathbb{E}\left(\mathcal{B}^{\prime \prime}\right)=\mathbb{E}\left(\mathcal{B}^{\prime}\right)$, we have

$$
\left|\operatorname{Cov}\left[\mathcal{A}^{\prime}, \mathcal{B}^{\prime}\right]-\operatorname{Cov}\left[\mathcal{A}^{\prime}, \mathcal{B}^{\prime \prime}\right]\right|=\left|\mathbb{E}\left[\mathbf{1}_{\mathcal{A}^{\prime}}\left(\mathbf{1}_{\mathcal{B}^{\prime}}-\mathbf{1}_{\mathcal{B}^{\prime \prime}}\right)\right]\right| \leq \operatorname{Pr}\left[\mathbf{1}_{\mathcal{B}^{\prime}} \neq \mathbf{1}_{\mathcal{B}^{\prime \prime}}\right]=O\left(n^{-1 / 2}\right),
$$

and thus,

$$
\operatorname{Cov}\left[\mathcal{A}^{\prime}, \mathcal{B}^{\prime}\right] \sim a^{2} / 2
$$

Comparing Equations (16) and (17), we see that (3) fails for $\left(\mathcal{A}^{\prime}, \mathcal{B}^{\prime}\right)$, as asserted.

Remark 3.3. Note that the family $\mathcal{B}^{\prime}$ in the example is not only regular but even fully symmetric. This shows that a symmetry assumption on only one of the families is insufficient. A weaker bound that does hold when one of the families is fully symmetric is Theorem 1.13.

Since the conclusion of Theorem 1.7 is not true in general, we can ask about the following weaker bound.

Statement 3.4. Let $\mathcal{A}, \mathcal{B} \subset \Omega_{n}$ be increasing. Then

$$
\operatorname{Cov}(\mathcal{A}, \mathcal{B}) \geq c \frac{\mathcal{W}_{1}\left(\mathbf{1}_{\mathcal{A}}, \mathbf{1}_{\mathcal{B}}\right)}{\log \left(e / \mathcal{W}_{1}\left(\mathbf{1}_{\mathcal{A}}\right) \mathcal{W}_{1}\left(\mathbf{1}_{\mathcal{B}}\right)\right)},
$$

where $c$ is a universal constant. 
Recall that Theorem 1.7 strengthens Theorem 1.3 by decreasing the denominator of the right hand side twice: First, it replaces $\mathcal{W}_{1}\left(\mathbf{1}_{\mathcal{A}}, \mathbf{1}_{\mathcal{B}}\right)$ inside the logarithm by $\sqrt{\mathcal{W}_{1}\left(\mathbf{1}_{\mathcal{A}}\right)} \sqrt{\mathcal{W}_{1}\left(\mathbf{1}_{\mathcal{B}}\right)}$, applying Cauchy-Schwarz. Second, it replaces the arithmetic mean $\log \left(e / \sqrt{\mathcal{W}_{1}\left(\mathbf{1}_{\mathcal{A}}\right)} \sqrt{\mathcal{W}_{1}\left(\mathbf{1}_{\mathcal{B}}\right)}\right)$ by the geometric mean $\sqrt{\log \left(e / \mathcal{W}_{1}\left(\mathbf{1}_{\mathcal{A}}\right)\right)} \sqrt{\log \left(e / \mathcal{W}_{1}\left(\mathbf{1}_{\mathcal{B}}\right)\right)}$. Statement 3.4 suggests to make only the first step.

While the families $(\mathcal{A}, \mathcal{B})$ of Example 3.1 satisfy Statement 3.4, the following example shows that Statement 3.4 is false, even under an additional assumption that one of the families is regular.

Example 3.2. For a small constant $a$, let $\mathcal{A}, \mathcal{B}$ be $\mathcal{A}=m_{a}\left(x_{1}, \ldots, x_{n}\right), \mathcal{B}=m_{1-a}\left(x_{1}, \ldots, x_{n}\right)$ as in Example 3.1, and let $\mathcal{C}=m_{1 / 2}\left(y_{1}, \ldots, y_{\ell}\right)$, where $\ell=\ell(n)$ is chosen such that $I_{i}(\mathcal{C})=I_{j}(\mathcal{B})$ for all $i, j$. Define $\mathcal{A}^{\prime}, \mathcal{B}^{\prime} \subset \Omega_{n+\ell}$ by $\mathcal{A}^{\prime}=\left\{\left(x_{1}, \ldots, x_{n}, y_{1}, \ldots, y_{\ell}\right):\left(\left(x_{1}, \ldots, x_{n}\right) \in \mathcal{A}\right) \vee\left(y_{1}=1\right)\right\}$ and $\mathcal{B}^{\prime}=\left\{\left(x_{1}, \ldots, x_{n}, y_{1}, \ldots, y_{\ell}\right):\left(\left(x_{1}, \ldots, x_{n}\right) \in \mathcal{B}\right) \wedge\left(y_{1}, \ldots, y_{\ell}\right) \in \mathcal{C}\right\}$. Note that by the choice of $\ell, \mathcal{B}^{\prime}$ is regular.

We claim that (18) fails for $\left(\mathcal{A}^{\prime}, \mathcal{B}^{\prime}\right)$. Indeed, a computation similar to that of Example 3.1 shows that $\operatorname{Cov}\left(\mathcal{A}^{\prime}, \mathcal{B}^{\prime}\right) \sim a^{2} / 4$, while $\mathcal{W}_{1}\left(\mathbf{1}_{\mathcal{A}}, \mathbf{1}_{\mathcal{B}}\right)=\Theta\left(a^{2} \log (1 / a)\right)$ and $\mathcal{W}_{1}\left(\mathbf{1}_{\mathcal{A}}\right)=\mathcal{W}_{1}\left(\mathbf{1}_{\mathcal{B}}\right)=$ $\Theta(1)$. Hence, the right hand side of (18) is $\Theta\left(a^{2} \log (1 / a)\right)$ which is significantly larger than the left hand side $\left(\Theta\left(a^{2}\right)\right)$, rendering (18) false.

Remark 3.5. We note that the same example, with $a=n^{-\alpha}$ for $\alpha \in(0,1 / 2)$, shows that in Theorem 1.13, the assumption that $\mathcal{B}$ is fully symmetric cannot be replaced by assuming that $\mathcal{B}$ is merely regular. Indeed, for $\left(\mathcal{A}^{\prime}, \mathcal{B}^{\prime}\right)$ of the example, the right hand side of (6) is

$$
c \sum_{i=1}^{n+\ell} \frac{I_{i}(\mathcal{A})}{\sqrt{\log \frac{e}{I_{i}(\mathcal{A})}}} \frac{I_{i}(\mathcal{B})}{\sqrt{\log \frac{e^{2}}{n I_{i}(\mathcal{B})^{2}}}}=\Theta\left(\frac{a^{2} \log (1 / a)}{\sqrt{\log n}}\right)=\Theta\left(a^{2} \cdot \alpha \sqrt{\log n}\right),
$$

which is asymptotically larger than $\operatorname{Cov}\left(\mathbf{1}_{\mathcal{A}^{\prime}}, \mathbf{1}_{\mathcal{B}^{\prime}}\right)=\Theta\left(a^{2}\right)$.

\section{An Asymmetric Correlation Bound}

In this section we present the proof of Theorem 1.13. This proof follows the original proof strategy of Talagrand [19], with a few enhancements that allow to handle in a better way different assumptions on $\mathcal{A}$ and $\mathcal{B}$. Due to this feature, we refer to the result as an "asymmetric" correlation bound.

\subsection{A few Lemmas}

In [19, the following simple lemma is proved and deployed.

Lemma 4.1. [19, Lemma 4.1] The function $\varphi(x)=\frac{x}{\log (e / x)}$ is increasing and convex in $(0,1)$, and for all $0<u \leq v<1$ we have

$$
\varphi(v) \leq \varphi(u)+\frac{2(v-u)}{\log (e / v)}
$$

We shall use Lemma 4.1, along with the following two strengthenings. 
Lemma 4.2. For any $n \in \mathbb{N}$, the function $\psi_{n}(x)=\frac{x}{\sqrt{\log \left(e^{3} / n x^{2}\right)}}$ is increasing and convex in $(0,1 / \sqrt{n})$, and for any $0<u \leq v<1 / \sqrt{n}$ we have

$$
\psi_{n}(v) \leq \psi_{n}(u)+\frac{2(v-u)}{\sqrt{\log \frac{e^{3}}{n((v+u) / 2)^{2}}}} .
$$

Lemma 4.3. The function $\psi(x)=x / \sqrt{\log \left(e^{2} / x\right)}$ is increasing and convex in $(0,1)$, and for any $0<u \leq v<1$ we have

$$
\psi(v) \leq \psi(u)+\frac{1.5(v-u)}{\sqrt{\log \frac{e^{2}}{(v+u) / 2}}} .
$$

We note that the main advantage of Lemmas 4.2 and 4.3 over Lemma 4.1 is replacement of $v$ by $(v+u) / 2$ in the denominator. This makes the proof of these lemmas a bit more complex than Talagrand's proof of Lemma 4.1. For sake of completeness, we present the proof of both lemmas below.

Proof of Lemma 4.2. We have

$$
\begin{aligned}
\psi_{n}^{\prime}(x) & =\left(\log \left(e^{3} / n x^{2}\right)\right)^{-1 / 2}+x \cdot(-1 / 2) \cdot\left(\log \left(e^{3} / n x^{2}\right)\right)^{-3 / 2} \cdot\left(n x^{2} / e^{3}\right) \cdot\left(-2 e^{3} / n x^{3}\right) \\
& =\left(\log \left(e^{3} / n x^{2}\right)\right)^{-1 / 2}\left(1+\left(\log \left(e^{3} / n x^{2}\right)\right)^{-1}\right)=h(x)\left(1+h(x)^{2}\right),
\end{aligned}
$$

where $h(x)=\log \left(e^{3} / n x^{2}\right)^{-1 / 2}$. As $h(x)$ is nonnegative and increasing in $(0,1 / \sqrt{n})$, it follows that $\psi_{n}^{\prime}$ is nonnegative and increasing, and thus $\psi_{n}$ is increasing and convex. Furthermore, we have $h(x) \leq 1$ and thus, $\psi_{n}^{\prime}(x) \leq 2 h(x)$. Hence,

$$
\psi_{n}(v)=\psi_{n}(u)+\int_{u}^{v} \psi_{n}^{\prime}(x) d x \leq \psi_{n}(u)+\int_{u}^{v} 2 h(x) d x .
$$

Now, we claim that $h(x)$ is concave in $(0,1 / \sqrt{n})$. Indeed, we have $h^{\prime}(x)=x^{-1} \log \left(e^{3} / n x^{2}\right)^{-3 / 2}$ and

$$
\begin{aligned}
h^{\prime \prime}(x) & =-x^{-2} \log \left(e^{3} / n x^{2}\right)^{-3 / 2}+x^{-1} \cdot 3 x^{-1} \log \left(e^{3} / n x^{2}\right)^{-5 / 2} \\
& =x^{-2} \log \left(e^{3} / n x^{2}\right)^{-3 / 2}\left(-1+3 \log \left(e^{3} / n x^{2}\right)^{-1}\right)<0,
\end{aligned}
$$

where the last inequality holds since $\log \left(e^{3} / n x^{2}\right)^{-1}<1 / 3$ for all $x \in(0,1 / \sqrt{n})$. Thus, by concavity of $h,(20)$ implies

$$
\psi_{n}(v) \leq \psi_{n}(u)+\int_{u}^{v} 2 h(x) d x \leq \psi_{n}(u)+2(v-u) h((v+u) / 2)=\psi_{n}(u)+\frac{2(v-u)}{\sqrt{\log \frac{e^{3}}{n((v+u) / 2)^{2}}}},
$$

as asserted.

Proof of Lemma 4.3. We have

$$
\begin{aligned}
\psi^{\prime}(x) & =\left(\log \left(e^{2} / x\right)\right)^{-1 / 2}+x \cdot(-1 / 2) \cdot\left(\log \left(e^{2} / x\right)\right)^{-3 / 2} \cdot\left(x / e^{2}\right) \cdot\left(-e^{2} / x^{2}\right) \\
& =\left(\log \left(e^{2} / x\right)\right)^{-1 / 2}\left(1+\frac{1}{2}\left(\log \left(e^{2} / x\right)\right)^{-1}\right)=h(x)\left(1+h(x)^{2} / 2\right)
\end{aligned}
$$


where $h(x)=\log \left(e^{2} / x\right)^{-1 / 2}$. As $h(x)$ is nonnegative and increasing in $(0,1)$, it follows that $\psi^{\prime}$ is nonnegative and increasing, and thus $\psi$ is increasing and convex. Furthermore, we have $h(x) \leq 1$ and thus, $\psi^{\prime}(x) \leq 1.5 h(x)$. Hence,

$$
\psi(v)=\psi(u)+\int_{u}^{v} \psi^{\prime}(x) d x \leq \psi(u)+\int_{u}^{v} 1.5 h(x) d x .
$$

Now, we claim that $h(x)$ is concave in $(0,1)$. Indeed, we have $h^{\prime}(x)=0.5 x^{-1} \log \left(e^{2} / x\right)^{-3 / 2}$ and

$$
\begin{aligned}
h^{\prime \prime}(x) & =0.5\left(-x^{-2} \log \left(e^{2} / x\right)^{-3 / 2}+x^{-1} \cdot 1.5 x^{-1} \log \left(e^{2} / x\right)^{-5 / 2}\right) \\
& =0.5 x^{-2} \log \left(e^{2} / x\right)^{-3 / 2}\left(-1+1.5 \log \left(e^{2} / x\right)^{-1}\right)<0,
\end{aligned}
$$

where the last inequality holds since $\log \left(e^{2} / x\right)^{-1}<1 / 2$ for all $x \in(0,1)$. Thus, by concavity of $h$, (21) implies

$$
\psi(v) \leq \psi(u)+\int_{u}^{v} 1.5 h(x) d x \leq \psi(u)+1.5(v-u) h((v+u) / 2)=\psi(u)+\frac{1.5(v-u)}{\sqrt{\log \frac{e^{2}}{((v+u) / 2)^{2}}}}
$$

as asserted.

Another simple but important lemma from [5, 19] (see also [16, Remark 5.28]) we use is the following:

Lemma 4.4. [19, Proposition 2.2] For any $f: \Omega_{n} \rightarrow[-1,1]$, with $\mathbb{E}[|f|] \leq 1 / 2$, we have

$$
\sum_{i=1}^{n} \hat{f}(\{i\})^{2} \leq c \mathbb{E}[|f|]^{2} \log (e / \mathbb{E}[|f|])
$$

where $c$ is an absolute constant.

As noted in [19], the following is an immediate corollary.

Corollary 4.5. For any $f: \Omega_{n} \rightarrow[-1,1]$, and for any $k$, we have

$$
\sum_{i \neq k} \hat{f}(\{i, k\})^{2} \leq c I_{k}(f)^{2} \log \left(e / I_{k}(f)\right)
$$

\subsection{Proof of Theorem 1.13}

A generalized statement of the theorem is the following.

Theorem. Let $f: \Omega_{n} \rightarrow[-1,1]$ be increasing and fully symmetric and $g: \Omega_{n} \rightarrow[-1,1]$ be increasing. Then

$$
\operatorname{Cov}(f, g) \geq c \sum_{i=1}^{n} \frac{I_{i}(f)}{\sqrt{\log \frac{e^{3}}{n I_{i}(f)^{2}}}} \frac{I_{i}(g)}{\sqrt{\log \frac{e^{2}}{I_{i}(g)}}}=\sum_{i=1}^{n} \psi_{n}\left(I_{i}(f)\right) \psi\left(I_{i}(g)\right),
$$

where $\psi_{n}(x)=\frac{x}{\sqrt{\log \left(e^{3} / n x^{2}\right)}}, \psi(x)=\frac{x}{\sqrt{\log \left(e^{2} / x\right)}}$, and $c$ is a universal constant.

Proof. The proof is by induction on $n$. The case $n=1$ is trivial, since in this case:

$$
\operatorname{Cov}(f, g)=I_{1}(f) I_{1}(g) \geq \psi_{1}\left(I_{1}(f)\right) \psi\left(I_{1}(g)\right) .
$$


We now prove the induction step. We choose to induct on the coordinate $j$ such that $I_{j}(g)=$ $\max _{i} I_{i}(g)$ and assume w.l.o.g. $j=n$. Define $f^{0}, f^{1}: \Omega_{n-1} \rightarrow[-1,1]$ by

$$
f^{0}\left(x_{1}, \ldots, x_{n-1}\right)=f\left(x_{1}, \ldots, x_{n-1}, 0\right) \quad \text { and } \quad f^{1}\left(x_{1}, \ldots, x_{n-1}\right)=f\left(x_{1}, \ldots, x_{n-1}, 1\right) .
$$

Denote by $a^{\ell}(\ell=0,1)$ the expectation $\mathbb{E}\left(f^{\ell}\right)$, by $a_{j}(j \in[n])$ the influence $I_{j}(f)$, and by $a_{j}^{\ell}$ $(\ell=0,1, j \in[n-1])$ the influence $I_{j}\left(f^{\ell}\right)$. Define $g^{0}, g^{1}, b^{\ell}, b_{j}, b_{j}^{\ell}$ in the same way, with $g$ in place of $f$. Since $f^{0}, f^{1}$ are fully symmetric, we have by the induction hypothesis

$$
\begin{aligned}
& \operatorname{Cov}\left(f^{0}, g^{0}\right)=\mathbb{E}\left[f^{0} g^{0}\right]-a^{0} b^{0} \geq c \sum_{i=1}^{n-1} \psi_{n-1}\left(a_{i}^{0}\right) \psi\left(b_{i}^{0}\right), \quad \text { and } \\
& \operatorname{Cov}\left(f^{1}, g^{1}\right)=\mathbb{E}\left[f^{1} g^{1}\right]-a^{1} b^{1} \geq c \sum_{i=1}^{n-1} \psi_{n-1}\left(a_{i}^{1}\right) \psi\left(b_{i}^{1}\right)
\end{aligned}
$$

Since $\mathbb{E}[f g]=\left(\mathbb{E}\left[f^{0} g^{0}\right]+\mathbb{E}\left[f^{1} g^{1}\right]\right) / 2$, we have

$$
\mathbb{E}[f g]-\left(a^{0} b^{0}+a^{1} b^{1}\right) / 2 \geq \frac{c}{2} \sum_{i=1}^{n-1}\left(\psi_{n-1}\left(a_{i}^{0}\right) \psi\left(b_{i}^{0}\right)+\psi_{n-1}\left(a_{i}^{1}\right) \psi\left(b_{i}^{1}\right)\right) .
$$

As $\mathbb{E}[f]=\left(a^{0}+a^{1}\right) / 2$ and $\mathbb{E}[g]=\left(b^{0}+b^{1}\right) / 2$, we obtain

$$
\operatorname{Cov}(f, g)=\mathbb{E}[f g]-\mathbb{E}[f] \mathbb{E}[g] \geq \frac{c}{2} \sum_{i=1}^{n-1}\left(\psi_{n-1}\left(a_{i}^{0}\right) \psi\left(b_{i}^{0}\right)+\psi_{n-1}\left(a_{i}^{1}\right) \psi\left(b_{i}^{1}\right)\right)+\frac{1}{4}\left(a^{1}-a^{0}\right)\left(b^{1}-b^{0}\right) .
$$

Note that $a^{1}-a^{0}=I_{n}(f)=a_{n}$ and $b^{1}-b^{0}=b_{n}$, and hence we actually have

$$
\operatorname{Cov}(f, g) \geq \frac{c}{2} \sum_{i=1}^{n-1}\left(\psi_{n-1}\left(a_{i}^{0}\right) \psi\left(b_{i}^{0}\right)+\psi_{n-1}\left(a_{i}^{1}\right) \psi\left(b_{i}^{1}\right)\right)+\frac{1}{4} a_{n} b_{n} .
$$

Thus, it is sufficient to show

$$
\frac{c}{2} \sum_{i=1}^{n-1}\left(\psi_{n-1}\left(a_{i}^{0}\right) \psi\left(b_{i}^{0}\right)+\psi_{n-1}\left(a_{i}^{1}\right) \psi\left(b_{i}^{1}\right)\right)+\frac{1}{4} a_{n} b_{n} \geq c \sum_{i=1}^{n} \psi_{n}\left(a_{i}\right) \psi\left(b_{i}\right)
$$

or equivalently,

$$
c \psi_{n}\left(a_{n}\right) \psi\left(b_{n}\right)+c \sum_{i=1}^{n-1}\left(\psi_{n}\left(a_{i}\right) \psi\left(b_{i}\right)-\frac{1}{2}\left(\psi_{n-1}\left(a_{i}^{0}\right) \psi\left(b_{i}^{0}\right)+\psi_{n-1}\left(a_{i}^{1}\right) \psi\left(b_{i}^{1}\right)\right)\right) \leq \frac{1}{4} a_{n} b_{n} .
$$

In the next steps, we consider the term $\psi_{n-1}\left(a_{i}\right) \psi\left(b_{i}\right)$ instead of $\psi_{n}\left(a_{i}\right) \psi\left(b_{i}\right)$, and we shall take care of the difference between them at a later stage. As for $\ell=0,1$ and for any $i \in[n-1]$, we have $(n-1)\left(a_{i}^{\ell}\right)^{2}=\sum_{i} I_{i}\left(f^{\ell}\right)^{2} \leq 1$ by Claim 2.4 (c), we can deduce $a_{i}^{\ell} \in(0,1 / \sqrt{n-1})$. Hence, we can use the convexity of $\psi_{n-1}$ and of $\psi$ (see Lemmas 4.2 and 4.3 ) to assert

$$
\psi_{n-1}\left(a_{i}\right) \psi\left(b_{i}\right)=\psi_{n-1}\left(\left(a_{i}^{0}+a_{i}^{1}\right) / 2\right) \psi\left(\left(b_{i}^{0}+b_{i}^{1}\right) / 2\right) \leq \frac{1}{4}\left(\psi_{n-1}\left(a_{i}^{0}\right)+\psi_{n-1}\left(a_{i}^{1}\right)\right)\left(\psi\left(b_{i}^{0}\right)+\psi\left(b_{i}^{1}\right)\right),
$$

for each $i \in[n-1]$. Thus,

$\psi_{n-1}\left(a_{i}\right) \psi\left(b_{i}\right)-\frac{1}{2}\left(\psi_{n-1}\left(a_{i}^{0}\right) \psi\left(b_{i}^{0}\right)+\psi_{n-1}\left(a_{i}^{1}\right) \psi\left(b_{i}^{1}\right)\right) \leq \frac{1}{4}\left(\psi_{n-1}\left(a_{i}^{0}\right)-\psi_{n-1}\left(a_{i}^{1}\right)\right)\left(\psi\left(b_{i}^{1}\right)-\psi\left(b_{i}^{0}\right)\right)$. 
Now, note that $a_{i}^{1}-a_{i}^{0}=2 \hat{f}(\{i, n\})$, and hence, by Lemma 4.2,

$$
\left|\psi_{n-1}\left(a_{i}^{0}\right)-\psi_{n-1}\left(a_{i}^{1}\right)\right| \leq \frac{2 \cdot 2 \hat{f}(\{i, n\})}{\sqrt{\log \frac{e^{3}}{(n-1)\left(\left(a_{i}^{0}+a_{i}^{1}\right) / 2\right)^{2}}}}=\frac{2 \cdot 2 \hat{f}(\{i, n\})}{\sqrt{\log \frac{e^{3}}{(n-1) a_{i}^{2}}}} .
$$

Similarly, $b_{i}^{1}-b_{i}^{0}=2 \hat{g}(\{i, n\})$, and hence, by Lemma 4.3.

$$
\left|\psi\left(b_{i}^{0}\right)-\psi\left(b_{i}^{1}\right)\right| \leq \frac{2 \cdot 2 \hat{g}(\{i, n\})}{\sqrt{\log \frac{e^{2}}{b_{i}}}} .
$$

Therefore, from (24) we get

$$
\sum_{i=1}^{n-1}\left(\psi_{n-1}\left(a_{i}\right) \psi\left(b_{i}\right)-\frac{1}{2}\left(\psi_{n-1}\left(a_{i}^{0}\right) \psi\left(b_{i}^{0}\right)+\psi_{n-1}\left(a_{i}^{1}\right) \psi\left(b_{i}^{1}\right)\right)\right) \leq \sum_{i=1}^{n-1} \frac{4|\hat{f}(\{i, n\}) \hat{g}(\{i, n\})|}{\sqrt{\log \frac{e^{3}}{(n-1) a_{i}^{2}}} \sqrt{\log \frac{e^{2}}{b_{i}}}} .
$$

In the denominator of the right hand side, we can replace $b_{i}$ by $b_{n}$ due to the choice of $n$. In the numerator, we replace $\sum_{i=1}^{n-1}|\hat{f}(\{i, n\}) \hat{g}(\{i, n\})|$ by

$$
\left(\sum_{i=1}^{n-1} \hat{f}(\{i, n\})^{2}\right)^{1 / 2}\left(\sum_{i=1}^{n-1} \hat{g}(\{i, n\})^{2}\right)^{1 / 2}
$$

using Cauchy-Schwarz, and bound the terms related to $f$ and the terms related to $g$ separately. For $g$, by Corollary 4.5 we have

$$
\sum_{i=1}^{n-1} \hat{g}(\{i, n\})^{2} \leq c_{1} b_{n}^{2} \log \left(e / b_{n}^{2}\right)
$$

and for $f$, by the full symmetry of $A$ we can use Lemma 2.7 to get

$$
\sum_{i=1}^{n-1} \hat{f}(\{i, n\})^{2}=\frac{2}{n} \mathcal{W}_{2}(f) \leq \frac{c}{n} \mathcal{W}_{1}(f) \log \left(e / \mathcal{W}_{1}(f)\right)=c_{2} a_{n}^{2} \log \left(e / n a_{n}^{2}\right) .
$$

Combining the bounds and summing over $i$, we obtain

$$
\left(\sum_{i=1}^{n-1} \hat{f}(\{i, n\})^{2}\right)^{1 / 2}\left(\sum_{i=1}^{n-1} \hat{g}(\{i, n\})^{2}\right)^{1 / 2} \leq \sqrt{c_{1} c_{2}} a_{n} b_{n} \sqrt{\log \frac{e}{n a_{n}^{2}}} \sqrt{\log \frac{e}{b_{n}}} .
$$

Substituting into (25) yields

$$
\begin{aligned}
\sum_{i=1}^{n-1}\left(\psi_{n-1}\left(a_{i}\right) \psi\left(b_{i}\right)-\frac{1}{2}\left(\psi_{n-1}\left(a_{i}^{0}\right) \psi\left(b_{i}^{0}\right)+\psi_{n-1}\left(a_{i}^{1}\right) \psi\left(b_{i}^{1}\right)\right)\right) & \leq \frac{4 \sqrt{c_{1} c_{2}} a_{n} b_{n} \sqrt{\log \frac{e}{n a_{n}^{2}}} \sqrt{\log \frac{e}{b_{n}}}}{\sqrt{\log \frac{e^{3}}{(n-1) a_{i}^{2}}} \sqrt{\log \frac{e^{2}}{b_{n}}}} \\
& \leq 4 \sqrt{c_{1} c_{2}} a_{n} b_{n} .
\end{aligned}
$$


As $\psi_{n}\left(a_{n}\right) \psi\left(b_{n}\right) \leq a_{n} b_{n}$, this almost proves (23), and thus the theorem. In order to complete the proof, we only have to "replace" $\psi_{n-1}\left(a_{i}\right)$ which we used in our argument with $\psi_{n}(a)$. This is done using the following calculation:

$$
\begin{aligned}
\left|\sum_{i=1}^{n-1} \psi_{n}\left(a_{i}\right) \psi\left(b_{i}\right)-\psi_{n-1}\left(a_{i}\right) \psi\left(b_{i}\right)\right| & \leq b_{n} \sum_{i=1}^{n-1}\left|\psi_{n}\left(a_{i}\right)-\psi_{n-1}\left(a_{i}\right)\right| \\
& \leq n a_{n} b_{n}\left(\left(\log \left(e^{3} / n a_{n}^{2}\right)\right)^{-1 / 2}-\left(\log \left(e^{3} /(n-1) a_{n}^{2}\right)\right)^{-1 / 2}\right) .
\end{aligned}
$$

Since for any $x, y>1$ we have $x^{-1}-y^{-1} \leq y-x \leq y^{2}-x^{2}$, we obtain

$$
\left(\left(\log \left(e^{3} / n a_{n}^{2}\right)\right)^{-1 / 2}-\left(\log \left(e^{3} /(n-1) a_{n}^{2}\right)\right)^{-1 / 2}\right) \leq \log \left(e^{3} /(n-1) a_{n}^{2}\right)-\log \left(e^{3} / n a_{n}^{2}\right)=\log \left(\frac{n}{n-1}\right) .
$$

Substituting into (27) yields

$$
\left|\sum_{i=1}^{n-1} \psi_{n}\left(a_{i}\right) \psi\left(b_{i}\right)-\psi_{n-1}\left(a_{i}\right) \psi\left(b_{i}\right)\right| \leq n a_{n} b_{n} \log \left(\frac{n}{n-1}\right) \leq 2 a_{n} b_{n} .
$$

Combining Equations (26) and (28), we obtain

$$
\sum_{i=1}^{n-1}\left(\psi_{n}\left(a_{i}\right) \psi\left(b_{i}\right)-\frac{1}{2}\left(\psi_{n-1}\left(a_{i}^{0}\right) \psi\left(b_{i}^{0}\right)+\psi_{n-1}\left(a_{i}^{1}\right) \psi\left(b_{i}^{1}\right)\right)\right) \leq\left(4 \sqrt{c_{1} c_{2}}+2\right) a_{n} b_{n},
$$

which implies that Equation (23) holds with $c=1 /\left(4 \sqrt{c_{1} c_{2}}+3\right)$, completing the proof.

Remark 4.6. We note that without the full symmetry assumption on $f$, we can use the same argument (using $\psi$ for both functions) to obtain an alternative proof of Theorem 1.4. The original proof presented in [14] is rather different, using a reduction from the Gaussian case and the so-called reverse isoperimetric inequality of Borell [4].

As demonstrated by Example 2.2 above, the full symmetry assumption on $f$ cannot be replaced by a regularity assumption. It will be interesting to find less restrictive sufficient conditions for Theorem 1.13 .

\section{Tightness of Theorem 1.3}

In this section we present several new tightness examples of Theorem 1.3. We present a few simple sufficient conditions and one necessary condition for tightness of (1), and then we give several concrete examples. Throughout the section, we use the notation $\mathbb{E}^{\prime}[h]=\min (\mathbb{E}[h], 1-$ $\mathbb{E}[h])$ for any $h: \Omega_{n} \rightarrow[0,1], \mathbb{E}^{\prime \prime}[h]=\min (1-\mathbb{E}[h], \mathbb{E}[h]+1)$ for any $h: \Omega_{n} \rightarrow[-1,1]$, and $\mu^{\prime}(\mathcal{C})=\min (\mu(\mathcal{C}), 1-\mu(\mathcal{C}))$ for any family $\mathcal{C}$.

\subsection{Conditions for tightness of Theorem 1.3}

We start with a simple necessary condition, which states that (11) can be tight only if the correlation of $f, g$ is rather small.

Proposition 5.1. Theorem 1.3 may be tight for $f, g: \Omega \rightarrow[0,1]$ only if $\operatorname{Cov}(f, g)=O\left(\mathbb{E}^{\prime}[f] \mathbb{E}^{\prime}[g]\right)$. 
Proof. Clearly, it is sufficient to prove that the right hand side of (11) is at most $O\left(\mathbb{E}^{\prime}[f] \mathbb{E}^{\prime}[g]\right)$. By Cauchy-Schwarz and the inequality between the arithmetic and geometric means, we have

$$
\begin{aligned}
\sum_{i=1}^{n} \frac{I_{i}(f) I_{i}(g)}{\log \left(e / \sum_{i=1}^{n} I_{i}(f) I_{i}(g)\right)} & =\frac{\mathcal{W}_{1}(f, g)}{\log \left(e / \mathcal{W}_{1}(f, g)\right)} \leq \frac{\sqrt{\mathcal{W}_{1}(f) \mathcal{W}_{1}(g)}}{0.5 \log \left(e^{2} / \mathcal{W}_{1}(f) \mathcal{W}_{1}(g)\right)} \\
& \leq \frac{\sqrt{\mathcal{W}_{1}(f) \mathcal{W}_{1}(g)}}{\sqrt{\log \left(e / \mathcal{W}_{1}(f)\right) \log \left(e / W_{1}(g)\right)}}=\sqrt{\varphi\left(\mathcal{W}_{1}(f)\right) \varphi\left(\mathcal{W}_{1}(g)\right)}
\end{aligned}
$$

where $\varphi(x)=x / \log (e / x)$ as above. By Lemma 4.4, we have $\mathcal{W}_{1}(f) \leq c \mathbb{E}^{\prime}[f]^{2} \log \left(e / \mathbb{E}^{\prime}[f]\right)$ and similarly for $g$. As $\varphi$ is increasing (Lemma 4.1), we obtain

$$
\sqrt{\varphi\left(\mathcal{W}_{1}(f)\right) \varphi\left(\mathcal{W}_{1}(g)\right)} \leq \sqrt{\varphi\left(c \mathbb{E}^{\prime}[f]^{2} \log \left(e / \mathbb{E}^{\prime}[f]\right)\right)} \sqrt{\varphi\left(c \mathbb{E}^{\prime}[g]^{2} \log \left(e / \mathbb{E}^{\prime}[g]\right)\right)} \leq c^{\prime} \mathbb{E}^{\prime}[f] \mathbb{E}^{\prime}[g],
$$

completing the proof.

Our first sufficient condition is also related to Lemma 4.4.

Notation 5.2. An increasing family $\mathcal{A} \subset \Omega_{n}$ is called first-level optimal if it is a tightness example (up to a constant) for Lemma 4.4, that is, if $\mathcal{W}_{1}\left(\mathbf{1}_{\mathcal{A}}\right) \geq c_{0} \mathbb{E}^{\prime}\left[\mathbf{1}_{\mathcal{A}}\right]^{2} \log \left(e / \mathbb{E}^{\prime}\left[\mathbf{1}_{\mathcal{A}}\right]\right)$ for a universal constant $c_{0}$. First-level optimality of a function $f: \Omega_{n} \rightarrow[-1,1]$ is defined similarly, with $\mathbb{E}^{\prime \prime}[f]$ in place of $\mathbb{E}^{\prime}\left[\mathbf{1}_{\mathcal{A}}\right]$.

As usual, the formally correct definition is to consider a family of families $\left\{\mathcal{A}_{m} \subset \Omega_{m}\right\}$, with an asymptotic property $\mathcal{W}_{1}\left(\mathbf{1}_{\mathcal{A}_{m}}\right)=\Omega\left(\mathbb{E}^{\prime}\left[\mathbf{1}_{\mathcal{A}_{m}}\right] \log \left(e / \mathbb{E}^{\prime}\left[\mathbf{1}_{\mathcal{A}_{m}}\right]\right)\right)$. For sake of simplicity, we treat a single family $\mathcal{A}=\mathcal{A}_{n}$ and assume that $n$ is sufficiently large.

Proposition 5.3. If $\mathcal{A}$ is first-level optimal and $\mathcal{B}$ is the dual of $\mathcal{A}$ then (1) is tight for $(\mathcal{A}, \mathcal{B})$ (up to the constant c).

Proof. We have to show that $\operatorname{Cov}(\mathcal{A}, \mathcal{B}) \leq c \varphi\left(\mathcal{W}_{1}\left(\mathbf{1}_{\mathcal{A}}, \mathbf{1}_{\mathcal{B}}\right)\right)$. Since $I_{i}(\mathcal{A})=I_{i}(\mathcal{B})$ for all $i$, we have

$$
\varphi\left(\mathcal{W}_{1}\left(\mathbf{1}_{\mathcal{A}}, \mathbf{1}_{\mathcal{B}}\right)\right)=\varphi\left(\mathcal{W}_{1}\left(\mathbf{1}_{\mathcal{A}}\right)\right) \geq \varphi\left(c_{0} \mathbb{E}^{\prime}\left[\mathbf{1}_{\mathcal{A}}\right] \log \left(e / \mathbb{E}^{\prime}\left[\mathbf{1}_{\mathcal{A}}\right]\right)\right) \geq c^{\prime} \mu^{\prime}(\mathcal{A}),
$$

the penultimate inequality using the first-level optimality of $\mathcal{A}$ and monotonicity of $\varphi$. On the other hand,

$$
\operatorname{Cov}(\mathcal{A}, \mathcal{B})=\mu(\mathcal{A} \cap \mathcal{B})-\mu(\mathcal{A}) \mu(\mathcal{B}) \leq \min (\mu(\mathcal{A}), \mu(B))=\mu^{\prime}(\mathcal{A})
$$

the last equality using $\mu(\mathcal{B})=1-\mu(\mathcal{A})$. Comparing (30) and (31) completes the proof.

The second sufficient condition is a simple composition lemma.

Notation 5.4. Let $f: \Omega_{n} \rightarrow \mathbb{R}$ and let $g_{1}, g_{2}, \ldots, g_{n}: \Omega_{m} \rightarrow\{0,1\}$. The composition $f \circ$ $\left(g_{1}, \ldots, g_{n}\right): \Omega_{m n} \rightarrow \mathbb{R}$ is defined by

$$
\begin{aligned}
f \circ\left(g_{1}, \ldots, g_{n}\right) & \left(x_{1}^{1}, \ldots, x_{m}^{1}, x_{1}^{2}, \ldots, x_{m}^{2}, \ldots, x_{1}^{n}, \ldots, x_{m}^{n}\right)= \\
& =f\left(g_{1}\left(x_{1}^{1}, \ldots, x_{m}^{1}\right), g_{2}\left(x_{1}^{2}, \ldots, x_{m}^{2}\right), \ldots, g_{n}\left(x_{1}^{n}, \ldots, x_{m}^{n}\right)\right) .
\end{aligned}
$$

Proposition 5.5. Let $\left(f_{1}, f_{2}\right)$, with $f_{1}, f_{2}: \Omega_{n} \rightarrow\{0,1\}$, be a tightness example for (11), and let $g_{1}, \ldots, g_{n}: \Omega_{m} \rightarrow\{0,1\}$ be increasing functions such that 
- $\mathbb{E}\left[g_{i}\right]=1 / 2$ for all $i$, and

- $\mathcal{W}_{1}\left(g_{i}\right) \geq c_{0}$ for all $i$, where $c_{0}$ is a universal constant.

Then $\left(f_{1} \circ\left(g_{1}, \ldots, g_{n}\right), f_{2} \circ\left(g_{1}, \ldots, g_{n}\right)\right)$ is also a tightness example for (1) (though, with a different constant).

Proof. For $\ell=0,1$, denote $\tilde{f}_{\ell}=\left(f_{\ell} \circ\left(g_{1}, \ldots, g_{n}\right)\right)$. It is clear that $\mathbb{E}\left[\tilde{f}_{\ell}\right]=\mathbb{E}\left[f_{\ell}\right]$, and $\mathbb{E}\left[\tilde{f}_{1} \tilde{f}_{2}\right]=$ $\mathbb{E}\left[f_{1} f_{2}\right]$ (here we use the fact that $g_{1}, \ldots, g_{n}$ are the same for $\left.f_{1}, f_{2}\right)$. Hence, $\operatorname{Cov}\left(\tilde{f}_{1}, \tilde{f}_{2}\right)=$ $\operatorname{Cov}\left(f_{1}, f_{2}\right)$. On the other hand, denoting by $I_{i, j}\left(f_{\ell}\right)$ the influence on the variable $x_{j}^{i}$ on $f_{\ell}$, we have $I_{i, j}\left(f_{\ell}\right)=I_{i}\left(f_{\ell}\right) I_{j}\left(g_{i}\right)$. Thus,

$$
\mathcal{W}_{1}\left(\tilde{f}_{1}, \tilde{f}_{2}\right)=\sum_{i} I_{i}\left(f_{1}\right) I_{i}\left(f_{2}\right) \sum_{j} I_{j}\left(g_{i}\right)^{2} \geq c_{0} \sum_{i} I_{i}\left(f_{1}\right) I_{i}\left(f_{2}\right)=c_{0} \mathcal{W}_{1}\left(f_{1}, f_{2}\right),
$$

where the inequality uses the assumption on $\left\{g_{i}\right\}$. Therefore, $\varphi\left(\mathcal{W}_{1}\left(\tilde{f}_{1}, \tilde{f}_{2}\right)\right) \geq c \varphi\left(\mathcal{W}_{1}\left(f_{1}, f_{2}\right)\right)$, completing the proof.

\subsection{A few properties of linear threshold functions}

Before we present the specific examples, we cite a few definitions and results on linear threshold functions that will be a central ingredient of the examples.

Definition 5.6. A linear threshold function is $f: \Omega_{n} \rightarrow\{-1,1\}$ of the form $f\left(x_{1}, \ldots, x_{n}\right)=$ $\operatorname{sign}\left(\sum a_{i} x_{i}-\theta\right)$, where $a_{i}, \theta \in \mathbb{R}$ and $\operatorname{sign}(x)=1$ if $x \geq 0$ and $\operatorname{sign}(x)=-1$ otherwise.

Linear threshold functions (LTFs) are a central object of study in computer science (see, e.g., [16]). It is clear that an LTF is increasing iff $a_{i} \geq 0$ for all $i$, and balanced (i.e., satisfies $\mathbb{E}[f]=0)$ iff $\theta=0$. The next definition captures the notion of low-influence functions.

Definition 5.7. A function $f: \Omega_{n} \rightarrow \mathbb{R}$ is called $\tau$-regular if $I_{i}(f) \leq \tau\|f\|_{2}$ for all $i$.

Intuitively, having low influences allows to approximate the function by a Gaussian via the Central Limit Theorem and to use Gaussian tools to handle it (see, e.g., [15]).

Notation 5.8. For $x \in(0,1)$, let $u(x)=2\left[\phi\left(\Phi^{-1}(x)\right)\right]^{2}$, where $\phi$ is the density function and $\Phi$ the cumulative distribution function of a Gaussian $N(0,1)$ random variable.

It is easy to see that if $x=1-\eta$, then $u(x)=\Theta\left(\eta^{2} \log (1 / \eta)\right)$ (see [15, Proposition 24]). We use the following theorem of Matulef et al. [15].

Theorem 5.9. ([15, Theorem 48]) Let $f_{1}\left(x_{1}, \ldots, x_{n}\right)=\operatorname{sign}\left(\sum a_{i} x_{i}-\theta_{1}\right)$ with $\sum a_{i}^{2}=1$ be $a$ $\tau$-regular LTF. Then

$$
\left|\mathcal{W}_{1}\left(f_{1}\right)-u\left(\mathbb{E}\left[f_{1}\right]\right)\right| \leq \tau^{1 / 6}
$$

Furthermore, if $f_{2}\left(x_{1}, \ldots, x_{n}\right)=\operatorname{sign}\left(\sum a_{i} x_{i}-\theta_{2}\right)$ is another LTF with the same weights $a_{i}$ then

$$
\left|\mathcal{W}_{1}\left(f_{1}, f_{2}\right)^{2}-u\left(\mathbb{E}\left[f_{1}\right]\right) u\left(\mathbb{E}\left[f_{2}\right]\right)\right| \leq \tau^{1 / 6} .
$$

An immediate corollary of Theorem 5.9 is that if $f$ is $\tau$-regular, where $\tau \leq c\left(\mathbb{E}^{\prime \prime}[f]^{2} \log \left(1 / \mathbb{E}^{\prime \prime}[f]\right)\right)^{6}$ for a sufficiently small $c$, then $f$ is first-level optimal.

For balanced LTFs, we can deduce the same conclusion without the $\tau$-regularity assumption, using the following theorem of Peres [17] (which shows that LTFs are asymptotically noise stable, see [2]): 
Theorem 5.10. 177 Let $f: \Omega_{n} \rightarrow\{-1,1\}$ be a balanced LTF. Then

$$
N S_{\epsilon}(f):=\frac{1}{2}-\frac{1}{2} \sum_{S \subset[n]}(1-2 \epsilon)^{|S|} \hat{f}(S)^{2} \leq O(\sqrt{\epsilon}) .
$$

Theorem 5.10 immediately implies that balanced LTFs are first-level optimal (using, e.g., [13, Theorem 4]).

\subsection{Specific examples}

Recall that there are two previously known examples: $\mathcal{A}$ being a small Hamming ball and $\mathcal{B}$ being its dual (presented by Talagrand) [19], and $\mathcal{A}$ being the tribes function and $\mathcal{B}$ being its dual [1].

Example 5.1. Our first example is an extension of Talagrand's example.

Proposition 5.11. Let $f$ be an increasing $\tau$-regular LTF, with $\tau \leq c\left(\mathbb{E}^{\prime \prime}[f]^{2} \log \left(1 / \mathbb{E}^{\prime \prime}[f]\right)\right)^{6}$ for a sufficiently small $c$. Let $\mathcal{A} \subset \Omega_{n}$ be a family such that $f=2 \cdot \mathbf{1}_{\mathcal{A}}-1$, and let $\mathcal{B}$ be the dual of $\mathcal{A}$. Then (11) is tight for $(\mathcal{A}, \mathcal{B})$.

Proof. As mentioned above, Theorem [5.9 implies that $\mathcal{A}$ is first-level optimal. The assertion now follows from Proposition 5.3 .

Talagrand's example is a special case, with $f=\operatorname{sign}\left(\sum_{i} \frac{1}{\sqrt{n}} x_{i}-\theta\right)$, for any $\theta$ such that $\mathbb{E}^{\prime \prime}[f]$ is not too small. It is plausible that Proposition 5.11 actually holds for any LTF (i.e., without the $\tau$-regularity assumption), which would yield a wider class of tightness examples.

Example 5.2. The second example is a generalization of a layered majority function with a constant number of layers. For simplicity of notation, we replace our domain $\Omega_{n}$ by $\Omega_{n}^{\prime}=$ $\{-1,1\}^{n}$.

Definition 5.12. A 1-layer weighted majority function is an increasing balanced LTF (on any number of coordinates, including a single coordinate). A k-layer weighted majority function is defined inductively as $f \otimes\left(g_{1}, g_{2}, \ldots, g_{n}\right)$, where $f: \Omega_{n}^{\prime} \rightarrow\{-1,1\}$ is an increasing balanced LTF and $g_{1}, \ldots, g_{n}$ are $k-1$-layer weighted majority functions.

Proposition 5.13. Let $k \in \mathbb{N}$ be constant, let $\left(f_{1}, f_{2}\right)$ be a pair of functions on $\Omega_{n}^{\prime}$ for which (1) is tight, and let $g_{1}, \ldots, g_{n}$ be layered majority functions with at most $k$ layers. Then (1) is tight for the functions $\left(f_{1} \circ\left(g_{1}, \ldots, g_{n}\right), f_{2} \circ\left(g_{1}, \ldots, g_{n}\right)\right)$.

Proof. As mentioned above, Theorem 5.10 implies that any balanced increasing LTF $g$ satisfies $\mathcal{W}_{1}(g) \geq c$. By induction on $k$, the same holds for any $k$-layer weighted majority (with a constant that depends on $k$ ). Since $k$ is assumed to be constant, the assertion follows from Proposition 5.5.

Example 5.3. The two example classes presented above consist of a family and its dual, as the previously known examples. A conceptually different type of examples is those presented in Section 3.2. For sake of completeness, we restate them here. 
Proposition 5.14. For a small constant a, let $\mathcal{A}=m_{a}\left(x_{1}, \ldots, x_{n}\right)$, and let $\mathcal{B}=m_{1-a}\left(x_{1}, \ldots, x_{n}\right)$ be the dual of $\mathcal{A}$. Then the following pairs are tightness examples for (11).

(a) $\mathcal{A}^{\prime}, \mathcal{B}^{\prime} \subset \Omega_{n+1}$, defined by

$$
\mathcal{A}^{\prime}=\left\{\left(x_{1}, \ldots, x_{n}, y\right):\left(\left(x_{1}, \ldots, x_{n}\right) \in \mathcal{A}\right) \vee(y=1)\right\}, \quad \text { and } \quad \mathcal{B}^{\prime}=m_{1-a}\left(x_{1}, \ldots, x_{n}, y\right) .
$$

(b) $\mathcal{A}^{\prime}, \mathcal{B}^{\prime} \subset \Omega_{n+\ell}$, defined by $\mathcal{A}^{\prime}=\left\{\left(x_{1}, \ldots, x_{n}, y_{1}, \ldots, y_{\ell}\right):\left(\left(x_{1}, \ldots, x_{n}\right) \in \mathcal{A}\right) \vee\left(y_{1}=1\right)\right\}$ and $\mathcal{B}^{\prime}=\left\{\left(x_{1}, \ldots, x_{n}, y_{1}, \ldots, y_{\ell}\right):\left(\left(x_{1}, \ldots, x_{n}\right) \in \mathcal{B}\right) \wedge\left(y_{1}, \ldots, y_{\ell}\right) \in \mathcal{C}\right\}$, where $\mathcal{C}=m_{1 / 2}\left(y_{1}, \ldots, y_{\ell}\right)$ and $\ell=\ell(n)$ is chosen such that $I_{i}(\mathcal{C})=I_{j}(\mathcal{B})$ for all $i, j$.

Proof. The tightness of (11) for both pairs of examples follows immediately from the computations presented in Section 3.2 .

Our concluding example is not a tightness example of Theorem 1.3, but rather provides a case study for comparing all lower bounds considered in the paper.

Example 5.4. Let $f_{1}=\operatorname{sign}\left(\sum a_{i} x_{i}-\theta\right)$ be a $\tau$-regular LTF, with $E[f]=1-a$ for a small constant $a$ and $\tau \leq c\left(\mathbb{E}^{\prime \prime}[f]^{2} \log \left(1 / \mathbb{E}^{\prime \prime}[f]\right)\right)^{12}$ for a sufficiently small $c$. Let $f_{2}=\operatorname{sign}\left(\sum a_{i} x_{i}\right)$.

Since $f_{1} f_{2}(x)=f_{2}(x)$ for all $x$, we have $\operatorname{Cov}\left[f_{1} f_{2}\right]=\mathbb{E}\left[f_{2}\right]-\mathbb{E}\left[f_{1}\right] \mathbb{E}\left[f_{2}\right]=a / 2$. On the other hand, by Theorem 5.9, we have

$$
\mathcal{W}_{1}\left(f_{1}, f_{2}\right)=\Theta(a \sqrt{\log (1 / a)}), \quad \mathcal{W}_{1}\left(f_{1}\right)=\Theta\left(a^{2} \log (1 / a)\right), \quad \text { and } \quad \mathcal{W}_{1}\left(f_{2}\right)=\Theta(1) .
$$

Hence, for the pair of functions $\left(f_{1}, f_{2}\right)$ the bound (3) is tight, while the bounds (11) and (18) are off by a factor of $\Theta(\sqrt{\log (1 / a)})$. In the specific case of $f_{1}$ corresponding to a Hamming ball, i.e., $f_{1}=\operatorname{sign}\left(\sum \frac{1}{\sqrt{n}} x_{i}-\theta\right)$, we can compute also the bounds (2) and (6) and find that (2) is off by a factor of $\log n / \sqrt{\log (1 / a)}$, while (6) is off by a factor of $\sqrt{\log (n) / \log (1 / a)}$.

This example demonstrates the advantage of Theorem 1.7 over all other bounds we consider. Note however that while Theorem 1.7 holds for $f_{1}=\operatorname{sign}\left(\sum \frac{1}{\sqrt{n}} x_{i}-\theta\right)$, we do not know whether it can be generalized to any low-influence LTF. We do know that it does not hold for LTFs in general (Example 3.1 being a counterexample), but it seems plausible that it should hold under an appropriate $\tau$-regularity assumption.

\section{Open Problems}

We conclude this paper with a few open problems.

Problem 6.1. A much stronger, and more "nice-looking", correlation lower bound is

$$
\operatorname{Cov}(f, g) \geq \sum_{i} I_{i}(f) I_{i}(g)
$$

It was shown in [11] that (32) holds "on average", i.e., when correlation is averaged over all pairs of elements in a family $\mathcal{T}$. While it clearly does not hold in general (all examples of Section 5 being counterexamples), it will be interesting to find additional conditions under which (32) holds, both for Boolean functions and for general functions. One condition that may be relevant is the submodularity condition which is of great interest in combinatorics and optimization. As shown in [9], for families of sets Equation (32), as well as several weaker correlation inequalities, are related to a conjecture of Chvátal in extremal set theory. 
Problem 6.2. It will be interesting to understand in which cases Lemma 4.4 is tight. That is, what are the families $\mathcal{A}$ that satisfy

$$
\sum_{i} I_{i}^{2}(\mathcal{A}) \geq c \mu(\mathcal{A})^{2} \log (1 / \mu(\mathcal{A}))
$$

for a universal constant $c$. This question seems to be of independent interest, due to the abundance of applications of Lemma 4.4, and also can provide more tightness examples for Theorem 1.3 (using Proposition [5.3). In [15], it is shown that if $\mathcal{W}_{1}(f)$ is very close to the maximum possible, then $f$ must be a linear threshold function. However, when we ask for tightness only up to a constant factor, the question looks harder. One specific case that may be easy to handle is to show that (33) holds for any LTF (and not only for low-influence LTFs as shown in Theorem 5.91).

Problem 6.3. It will be interesting to find additional conditions under which Theorem 1.7 holds, i.e.,

$$
\operatorname{Cov}(\mathcal{A}, \mathcal{B}) \geq c \frac{\mathcal{W}_{1}\left(\mathbf{1}_{\mathcal{A}}, \mathbf{1}_{\mathcal{B}}\right)}{\sqrt{\log \left(e / \mathcal{W}_{1}\left(\mathbf{1}_{\mathcal{A}}\right)\right)} \sqrt{\log \left(e / \mathcal{W}_{1}\left(\mathbf{1}_{\mathcal{B}}\right)\right.}}
$$

for a universal constant $c$. In particular, it seems plausible that (34) holds for any pair of low-influence LTFs. If true, this will provide an additional tightness example of Theorem 1.7, in a case where all other bounds considered in this paper are not tight (see Example 4.4.).

\section{Acknowledgements}

We are grateful to Ryan o'Donnell for suggesting to use Theorem 5.9 to provide tightness examples for Lemma 4.4.

\section{References}

[1] D. Ahlberg, E. Broman, S. Griffiths, and R. Morris, Noise sensitivity in continuum percolation, Israel J. Math., 201(2) (2014), pp. 847-899.

[2] I. Benjamini, G. Kalai, and O. Schramm, Noise sensitivity of Boolean functions and applications to percolation, Inst. Hautes Etudes Sci. Publ. Math. 90 (1999), pp. 5-43.

[3] M. Ben-Or and N. Linial, Collective coin flipping, in Randomness and Computation (S. Micali, ed.), Academic Press, New York, 1990, pp. 91-115.

[4] C. Borell, Positivity improving operators and hypercontractivity, Math. Zeitschrift, 180(2) (1982), pp. 225-234.

[5] M.-C. Chang, A polynomial bound in Freiman's theorem, Duke Math. J. 113(3), pp. 399419, 2002.

[6] V. Chvátal, Intersecting families of edges in hypergraphs having the hereditary property. Hypergraph Seminar (Proc. First Working Sem., Ohio State Univ., Columbus, Ohio, 1972; dedicated to Arnold Ross), pp. 61-66. Lecture Notes in Math., Vol. 411, Springer, Berlin, 1974.

[7] Y. Filmus, H. Hatami, N. Keller, and N. Lifshitz, Bounds on the sum of $L_{1}$ influences, Israel J. Math., to appear. 
[8] C.M. Fortuin, P.W. Kasteleyn, and J. Ginibre, Correlation inequalities on some partially ordered sets, Comm. Math. Phys. 22 (1971), pp. 89-103.

[9] E. Friedgut, J. Kahn, G. Kalai, and N. Keller, Chvátal's conjecture and correlation inequalities, preprint, 2015.

[10] T.E. Harris, A lower bound for the critical probability in a certain percolation process, Proc. Cambridge Phil. Soc. 56 (1960), pp. 13-20.

[11] N. Keller, Lower bound on the correlation between monotone families in the average case, Adv. Appl. Math., 43(1) (2009), pp. 31-45.

[12] N. Keller, On the probability of a rational outcome for generalized social welfare functions on three alternatives, J. Combin. Th. Ser. A, 117(4) (2010), pp. 389-410.

[13] N. Keller and G. Kindler, Quantitative relation between noise sensitivity and influences, Combinatorica, 33(1) (2013), pp. 45-71.

[14] N. Keller, E. Mossel, and A. Sen, Geometric influences II: Correlation inequalities and noise sensitivity, Ann. Inst. Henri Poincare 50(4), pp. 1121-1139, 2014.

[15] K. Matulef, R. O'Donnell, R. Rubinfeld, and R. A. Servedio, Testing halfspaces, SIAM J. Comput. 39(5) (2010), pp. 2004-2047.

[16] R. O’Donnell, Analysis of Boolean functions, Cambridge University Press, 2014.

[17] Y. Peres, Noise stability of weighted majority, manuscript, 2004. Available at: arXiv:math/0412377.

[18] L. Russo, A Note on percolation, Z. Wahrscheinlichkeitstheorie und Verw. Gebiete 43(1) (1978), pp. 39-48.

[19] M. Talagrand, How much are increasing sets positively correlated?, Combinatorica 16 (1996), no. 2, pp. 243-258.

[20] M. Talagrand, On boundaries and influences, Combinatorica 17 (1997), no. 2, pp. 275-285. 